\title{
A Review of Hybrid Brain-Computer Interface Systems
}

\author{
Setare Amiri, Reza Fazel-Rezai, and Vahid Asadpour \\ Biomedical Signal and Image Processing Laboratory, Department of Electrical Engineering, University of North Dakota, \\ Grand Forks, ND, USA \\ Correspondence should be addressed to Reza Fazel-Rezai; Reza.Fazel-Rezai@engr.und.edu
}

Received 7 July 2012; Revised 30 October 2012; Accepted 4 December 2012

Academic Editor: Dimitrios Pantazis

Copyright (c) 2013 Setare Amiri et al. This is an open access article distributed under the Creative Commons Attribution License, which permits unrestricted use, distribution, and reproduction in any medium, provided the original work is properly cited.

\begin{abstract}
Increasing number of research activities and different types of studies in brain-computer interface (BCI) systems show potential in this young research area. Research teams have studied features of different data acquisition techniques, brain activity patterns, feature extraction techniques, methods of classifications, and many other aspects of a BCI system. However, conventional BCIs have not become totally applicable, due to the lack of high accuracy, reliability, low information transfer rate, and user acceptability. A new approach to create a more reliable BCI that takes advantage of each system is to combine two or more BCI systems with different brain activity patterns or different input signal sources. This type of BCI, called hybrid BCI, may reduce disadvantages of each conventional BCI system. In addition, hybrid BCIs may create more applications and possibly increase the accuracy and the information transfer rate. However, the type of BCIs and their combinations should be considered carefully. In this paper, after introducing several types of BCIs and their combinations, we review and discuss hybrid BCIs, different possibilities to combine them, and their advantages and disadvantages.
\end{abstract}

\section{Introduction}

A brain-computer interface (BCI) system can provide a communication method to convey brain messages independent from the brain's normal output pathway [1]. Brain activity can be monitored using different approaches such as standard scalp-recording electroencephalogram (EEG), magnetoencephalogram (MEG), functional magnetic resonance imaging (fMRI), electrocorticogram (ECoG), and near infrared spectroscopy (NIRS) [1-4]. However, EEG signals are considered as the input in most BCI systems. In this case, BCI systems are categorized based on the brain activity patterns such as event-related desynchronization/synchronization (ERD/ERS), steady-state visual evoked potentials (SSVEPs), P300 component of event related potentials (ERPs), and slow cortical potentials (SCPs) [5-16]. Each BCI type has its own shortcoming and disadvantages. To utilize the advantages of different types of BCIs, different approaches are combined, called hybrid BCIs $[15,16]$. In a hybrid BCI, two types of BCI systems can be combined. It is also possible to combine one BCI system with another system which is not BCI-based, for example, combining a BCI system with an electromyogram (EMG)-based system. However, one can debate if this type of system should be defined as hybrid BCI. In the rest of this paper, we assume that if an EEG BCI system is combined with another physiological signal (e.g., EMG) based system, a hybrid BCI system will be constructed.

Although different BCI methods can be combined, it should be noted that not all combinations of different brain imaging methods are feasible and possible. One of the limiting factors is the technology. For example, although MEG is a very high resolution brain imaging technique, it is not practical to use it when subjects need to move around. In addition, different techniques and their combinations should be utilized based on the application that the hybrid BCI is going to be used for. The main purpose of combining different systems to form a "hybrid" BCI is to improve accuracy, reduce errors, and overcome disadvantages of each conventional BCI system. Different types of hybrid BCI systems can be defined according to the types of systems which are combined, how systems are joined, and what types of inputs are considered. In non-hybrid BCIs, based on the property of EEG signals used as the input of BCI system, four major EEG-based BCIs are considered: SSVEP, P300, SCP, and ERD/ERS. 
Evoked response in EEG signals to repetitive visual stimulations is called SSVEP. In a SSVEP BCI paradigm, specific frequencies are allocated to the repetitive stimuli. For SSVEP detection, the frequency spectrum of the EEG is computed. Around the frequency of the repetition of stimulus in which the subject focuses, there will be peak on the frequency spectrum. By detecting this frequency, an intention of the subject can be detected. This can be translated to a control signal for a BCI system. There are some issues about SSVEP BCIs, one is gaze dependence $[9,17]$. Another issue is that in some users, the flickering stimulus is annoying and produces fatigue. Using higher frequencies for the flickering stimuli reduces the annoyance, but on the other hand, it is harder to detect the SSVEP. SSVEP BCIs have some advantages such as no significant training requirement and high information transfer rate $[6,18,19]$.

In P300-based BCIs, intention of the subjects is measured using the P300 component of the brain evoked response [20]. After stimulus onset, positive and negative deflections occur in the EEG. These deflections are called event-related potential (ERP) components. Depending on the latency of these deflections, they are grouped as "exogenous components" and "endogenous components" [10]. The exogenous components occur until about $150 \mathrm{msec}$ after the eliciting stimulus. The endogenous components have longer latency. The largest positive deflection that occurs between 250 to $750 \mathrm{msec}$ after the stimulus onset is called "P300". The P300 component is the most used ERP component in BCI systems. The paradigm that elicits P300 is called the "oddball paradigm" [21]. In an oddball paradigm, events that elicit the P300 fall into two classes in which one of the classes is less frequent. Interstimulus interval time and the frequency of the oddball stimulus are among the parameters that determine the amplitude of the P300 component. The first BCI P300-based system was introduced by Farwell and Donchin for spelling characters in 1988 [13].

Slow negative voltage shifts that occur in the EEG recorded over sensorimotor cortex, while actual or imagined movement happens [9] are called SCP. SCP-based BCI consists of series of trials [22]. Early SCP BCIs were especially slow, since in each trial only one selection was possible and the time needed for each selection was at least $10 \mathrm{sec}$. The temporal efficiency was improved by Kübler et al. to $4 \mathrm{sec}$ [23]. Shortening the time process further was not possible because users were uncomfortable with the shortened trial time. Over the past decade studies about the SCP approach have been limited because of several SCP BCI problems, which reduce the applicability of this type of BCI. Among others, SCP BCIs have three main problems: poor multidimensional control, high probability of error, and long-term training.

Rhythmic activity of EEG in terms of event-related desynchronization/synchronization (ERD/ERS) has been used as one of the sources in BCI [1]. Motor imagery is one way to induce changes in ERD/ERS and has been used in many BCI systems [24]. During motor imagery of movements, ERD occurs predominantly over the contralateral brain motor area and, therefore, can be used as a signal for a BCI system. ERD/ERS BCIs have been used in different applications such as achieving two-dimensional cursor control.

\section{Hybrid BCI Systems}

In recent years, there has been more attention to hybrid BCI systems. Based on Scopus search engine [25] and keyword (("Brain-Computer Interface" or "BCI") and "Hybrid"), the number of journal papers found before 2010 was only three. This number was 6 and 10 for 2010 and 2011, respectively. This shows increased attention to hybrid BCIs in the recent years.

In general, in a hybrid BCI, two systems can be combined sequentially or simultaneously [26]. In a simultaneous hybrid BCI, both systems are processed in parallel. Input signals used in simultaneous hybrid BCIs can be two different brain signals, one brain signal, or one brain signal and another input. In sequential hybrid BCIs, the output of one system is used as the input of the other system. This approach is mostly used when the first system task is to indicate that the user does not intend to communicate or as a "brain switch" [26].

\section{Review of Different Hybrid BCIs}

The combinations of the BCI types and a summary of important features of different hybrid BCIs which are discussed and reviewed in this paper are shown in Table 1.

3.1. SSVEP-Motor Imagery Hybrid BCI. In order to acquire better understanding about a hybrid based on SSVEP and motor imagery BCI, short summaries of approaches are mentioned as follows. In [15], the proposed hybrid was evaluated during the task and was applied under three conditions: ERD BCI, SSVEP BCI, and ERD-SSVEP BCI. During the ERD BCI task, two arrows appeared on the screen. When the left arrow appeared, subjects were instructed to imagine opening and closing their left hand. For the right arrow, subjects imagined opening and closing the corresponding hand. In the SSVEP task, subjects were instructed to gaze at either left $(8 \mathrm{~Hz})$ or right $(13 \mathrm{~Hz})$ LED depending on which cue appeared. In the hybrid task, when the left arrow was showed, subjects were imagining the left hand opening and closing while gazing at the left LED simultaneously. The task was similar for the right arrow. Results show the average accuracy of $74.8 \%$ for ERD, 76.9\% for SSVEP, and $81.0 \%$ for hybrid. The number of illiterate subjects, who achieved less than 70\% accuracy [38], reduced to zero from five using the hybrid approach.

A hybrid SSVEP/ERD BCI was introduced in [16] for orthosis control application. The SSVEP-based BCI was utilized for opening the orthosis at the activating stage, and an ERS-based BCI was used as a switch to deactivate the LEDs that were mounted on the orthosis for SSVEP evocation in the resting stage. The SSVEP-based stage entails four steps for opening and closing the orthosis completely. Frequencies 8 and $13 \mathrm{~Hz}$ LEDs were used for the opening and closing tasks, respectively. During training sessions, subjects were instructed to close the brain switch. Then, they were instructed to open and close the orthosis by gazing at the LEDs mounted on the orthosis. In the next stage, the SSVEPbased BCI was turned off by opening the brain switch. This switch was kept open during the resting period. At the end of the resting period, the brain switch was closed, and SSVEP task was repeated. After this experiment, subjects undertook 
TABLE 1: A comparison of several different BCI hybrid systems.

\begin{tabular}{|c|c|c|c|c|c|}
\hline Paper \# & Hybrid type & System organization & Improvement & Number of subjects & Classification \\
\hline$[15]$ & ERD, SSVEP & Simultaneous & $\begin{array}{l}\text { Accuracy significantly improved compared to } \\
\text { ERD and slightly better than SSVEP }\end{array}$ & 14 & LDA \\
\hline$[16]$ & ERD, SSVEP & Sequential & False positive rate was reduced & 6 & FLDA \\
\hline [27] & ERD, SSVEP & Sequential & $\begin{array}{l}\text { Application of BCI for FES triggering was } \\
\text { improved }\end{array}$ & 3 & $\begin{array}{l}\text { Filters and } \\
\text { thresholds }\end{array}$ \\
\hline [28] & ERD, SSVEP & Simultaneous & Feedbacks were added to the work done in [15] & 12 & LDA \\
\hline$[29]$ & P300, SSVEP & Sequential & Improved ITR & 10 & FLDA and BLDA \\
\hline$[30]$ & P300, SSVEP & Sequential & New application (smart home) & 3 & LDA \\
\hline$[31]$ & P300, ERD & Sequential & Improvement in application (wheelchair control) & 2 & Frequency analysis \\
\hline$[32]$ & P300, ERD & Sequential & Expand control functions in virtual environment & 4 & SVM and FLDA \\
\hline [33] & P300, ERD & Simultaneous & Increase reliability & 4 & $\begin{array}{c}\text { Fisher's } \\
\text { discriminant } \\
\text { analysis }\end{array}$ \\
\hline$[34]$ & ERD, NIRS & Simultaneous & $\begin{array}{l}\text { Improvement in classification accuracy and } \\
\text { performance }\end{array}$ & 14 & LDA \\
\hline$[35]$ & EEG, EMG & Simultaneous & Improvement in performance & 12 & $\begin{array}{c}\text { Frequency analysis } \\
\text { and Gaussian } \\
\text { classifier }\end{array}$ \\
\hline [36] & ERD, EOG & Simultaneous & $\begin{array}{l}\text { Improvement in classification accuracy, reduction } \\
\text { in number of electrodes and training time }\end{array}$ & 3 & Frequency analysis \\
\hline [37] & ERD, EOG & Sequential & Improvement in performance & 7 & LDA \\
\hline
\end{tabular}

the SSVEP-based BCI task alone and the LEDs were flickering during the resting period. For SSVEP detection, the power density spectrum was used. For the activity period, the true positive rate and false positive rate were measured and for resting period, the false positive rate. It was shown that false positive rate was reduced by more than $50 \%$ when hybrid BCI was utilized.

SSVEP and ERD were combined in [27] to make a twostage hybrid BCI system for triggering a Functional Electrical Stimulation (FES) system. In the first stage, SSVEP was presented for object selection. For evoking SSVEP, three LEDs with 15, 17 and $19 \mathrm{~Hz}$ frequencies were considered. The EEG was acquired from $\mathrm{O} 1, \mathrm{O} 2$, and $\mathrm{Oz}$ channels while considering $\mathrm{Cz}$ as a reference. The object selection task represented three basic grasps: palmar, lateral and precision grasp. For the analysis, Oz channel was chosen as the SSVEP activity in this channel was more noticeable compared to other channels. For SSVEP detection, Butterworth's band pass filters were used to separate frequency bands and a threshold for each subject was fixed manually. After selecting one of the three grasp options based on SSVEP, the next task was reaching movement in which ERD-based BCI was used. EEG signals for this task were recorded from the C3 channel. The $\mathrm{Cz}$ channel was used as the reference point. The signal was filtered utilizing Butterworth's band pass filters. The detection algorithm was based on the real-time mu and beta band-power estimation. The signal was compared with the manual adjusted threshold and a drop under the threshold was considered as a movement command. 98.1\% accuracy was achieved in the SSVEP stage. Using mu and beta bands, $100 \%$ and $98.1 \%$ accuracy were achieved, respectively. This study showed that the presented hybrid BCI can be considered as one of the appropriate combinations for FES triggering application.

In general, hybrid BCIs are more complex and depending on the types of the BCIs, the difficulty may be increased and user acceptability may be decreased. The user acceptability can be measured based on a questionnaire from the subjects. In [28], subjects found the hybrid BCI slightly more difficult than non-hybrid BCIs. In [28], ERD and SSVEP were combined for a simultaneous hybrid task. Bipolar channels $\mathrm{C} 3, \mathrm{Cz}, \mathrm{C} 4, \mathrm{O} 1$, and $\mathrm{O} 2$ were utilized for EEG recording. After training sessions, in the online run for SSVEP task, a cue pointed to the top LED, which was flickering with $8 \mathrm{~Hz}$ and then pointed to the bottom $13 \mathrm{~Hz}$ LED. Subjects received a real-time feedback from a rectangular appearing on the screen. During the ERD task, a cue pointed to the top of the screen and subjects imagined the opening and closing of both hands. When the cue pointed to the bottom of screen, subjects were instructed to imagine moving both feet. In the hybrid condition, both tasks were done simultaneously. The data from the training sessions was used for setting up the LDA classifier. The cross-validation classification accuracy was calculated for both online and the training sessions. In the training sessions, mean classification accuracy was $79.9 \%$, 98.1\%, and $96.5 \%$ for ERD, SSVEP, and hybrid condition, respectively. The analysis of the online performance showed that the mean classification accuracy was $76.9 \%, 94 \%$, and 95.6\% for ERD, SSVEP, and hybrid condition. For the same conditions maximum ITR was 3.2, 6.1, and 6.3 bits per min. In another analysis, the ERD and SSVEP features were classified separately in the hybrid BCI which showed that subjects were not doing only one of the tasks. Based on a questionnaire, two subjects indicated that hybrid BCI was much more difficult 
and their performance declined compared to the SSVEP condition. Four subjects indicated that there was not any difference in difficulty of the hybrid condition compared to two other conditions and their performance stayed the same or improved in the hybrid condition. Overall from the questionnaire, the hybrid condition was moderately more difficult. Comparing the results of this experience with the previous one [15], improvement was seen in the ERD results as the performance of the task had been changed (right hand versus left hand movement imagination in the previous study and both hand versus both feet movement imagination in this study). Other results, such as the lower accuracy in ERD condition and the higher performance in SSVEP condition, were consistent. The accuracy in the hybrid BCI is not significantly different from the SSVEP condition. By changing the classification or the combinations of the features, improvement in results may appear. However, the reliability of the system is improved as the SSVEP BCI is added to the conventional ERD BCI system. For subjects with low performance with ERD, or in the case of fatigue, the SSVEP BCI is appropriate option.

3.2. P300-SSVEP Hybrid BCI. P300 and SSVEP BCI were introduced as hybrids in an asynchronous BCI system in [29]. It seems the P300 and SSVEP combination worked well as the stimuli for evoking both patterns, which can be shown on one screen simultaneously. The P300 paradigm considered in this study is a $6 \times 6$ speller matrix based on the original P300 row/column paradigm introduced by Farwell and Donchin [13]. Only one frequency is allocated for the SSVEP paradigm. The background color was flashed with a frequency slightly less than $18 \mathrm{~Hz}$. The background color change facilitates the SSVEP detection. During the classification, P300 and SSVEP signals were separated by a band pass filter. The SSVEP was utilized as a control state (CS) detection. When the user was gazing at the screen, the SSVEP was detected and it was assumed that the user intended to send a command. The system detected the P300 target selection and CS simultaneously.

For SSVEP detection, the mean power spectral density (PSD) in the narrow band near the desired frequency and the PSD in the wider range near the desired frequency were utilized in an objective function. These values were subtracted from each other and divided over the PSD value from the wide band and the function value was compared to a specified threshold. During the data acquisition, the channels for acquiring EEG signals were not fixed for all subjects. For P300 classification, Bayesian linear discriminant analysis (BLDA) or Fischer's linear discriminant analysis (FLDA) were utilized $[39,40]$. The experiment was presented as an offline and online test. Ten subjects participated in the experiment. In the offline test, forty characters were presented for detection, which were divided into four groups. For better evaluation, SSVEP was presented only to two groups out of four groups. In CS, subjects were instructed to count the number of times they distinguished the highlighted character. In the non-control state (NCS), subjects were instructed to do a mental task like multiplication of two numbers and relax with closed eyes. For four out of five subjects, the accuracy was improved insignificantly during the presence of SSVEP and P300 detection was not determinate. Between the ten character's detection, there was a break of a certain time, which was due to the subject pressing a keyboard button. When the NCS time was almost finished, an auditory cue alerted subjects. For P300 an average classification accuracy of $96.5 \%$ and control state detection accuracy of $88 \%$ with the information transfer rate (ITR) of $20 \mathrm{bits} / \mathrm{min}$ were achieved during the offline test. The online test was presented under a semi synchronous condition. The experiment consisted of blocks with five rounds, for detecting each character. SSVEP detection for at least three out of five runs showed the control state detection by the subject, and P300 was detected during the control state. If the control state was not detected, the "=" character would be shown on the screen. The break time and the auditory alert were the same as the offline test. An average control state detection accuracy of $88.15 \%$, a classification accuracy of $94.44 \%$, and an ITR of $19.05 \mathrm{bits} / \mathrm{min}$ were achieved during the online test.

P300 and SSVEP combination was also introduced to control smart home environments in [30]. P300-based BCI was used for controlling the virtual smart home environment and SSVEP was implemented as a toggle switch for the P300 BCI operation. Results from this experiment show that P300 is suitable for discrete control commands and SSVEP is suitable for continuous control signals. The hybrid BCI achieved high accuracy and reliability in all subjects.

3.3. P300-Motor Imagery Hybrid BCI. Another possible combination for a hybrid $\mathrm{BCI}$ is $\mathrm{P} 300$ and motor imagery (MI)based BCI [31-33]. The basic concept in this type of hybrid is based on the features of P300 and ERD/ERS in control applications. P300 is a reliable BCI type for selecting one item out of several items and can be used for discrete control commands. On the other hand, due to the low degree of freedom presented by MI-based $\mathrm{BCI}$, this type of $\mathrm{BCI}$ is more efficient for continuous control commands. These two types of BCIs can be joined to present more complicated control commands in one task.

In [31], for controlling a wheelchair in a home environment, several approaches using different BCI techniques were introduced. The wheelchair control commands were divided into three steps.

Step 1 (Destination Selection). In this task, the user should select the destination of the wheelchair motion by selecting one of the items among a list of destinations. To implement this control command, an accurate and reliable interface is needed and false acceptance rates should be as low as possible. For this task, a P300 BCI presented at a screen was utilized. The experiments on healthy subjects showed a response time of about 20 seconds, the false acceptance rate $2.5 \%$ and the error less than $3 \%$. The results showed that P300 was an appropriate option for the interface. But there are a couple of points to be considered: First, all subjects were healthy. For users with severe disability, the accuracy of the results may differ. Second, there is concern about the applicability of the interface, if it is proper for daily use. A more applicable situation should be considered for evaluating this approach. 
Step 2 (Navigation). An autonomous motion control was introduced for this step. The destination was selected and the wheelchair started its motion toward the destination following virtual guiding paths. A proximity sensor was considered for stopping the wheelchair facing obstacles.

Step 3 (Stopping Command). For this control command, the interface needs to be fast, reliable and have a low false acceptance rate. Two approaches for a stopping command were presented. The first approach was the fast P300, in which, on the screen, there is only one item "The Stop" and the task is the detection of user's intention. Experimental results showed reduction in response time. However, increase in false acceptance makes this approach inapplicable. The second approach was to use a mu-beta BCI. The position of a cursor was considered for presenting the visual feedback for the mu-beta BCI system and the control of the cursor was based on an arm movement imagination. Results showed approximately the same response time as the fast P300 approach, but for false acceptance, a rate of zero was achieved. Since the low false acceptance rate and fast response are the most important needs for this type of BCI, it seems that mubeta BCI is a more reliable system for this application.

In [32], different states and control commands needed for operating the system were controlled in a virtual environment. P300/MI hybrid BCI was used for operating the system. Two sequential states covered the areas of the virtual environment, navigation, and device control state. The interface strategy is explained as follows. For navigation, MI BCI was used with the continuous control commands limited number of commands. By imagination of left and right hand movement, control commands were issued. The position in the virtual environment was updated by each control command.

In the device control state, the commands were discrete. By considering features of control commands and paradigms, an interface was developed. For this paradigm, the P300 oddball paradigm was considered. When the area coverage changed to the device control state, the MI command detection stopped and the controller switched to system state. The system state then switched to device control state automatically. The P300 BCI presented the control panel to subjects. A switch for navigation happened by the selection of the "quit" command using the P300 oddball paradigm. If the "quit" was not detected after 6 commands, the controller would switch to the system state automatically.

Experiments were performed by four subjects. To evaluate the hybrid approach, the experiment was also implemented for P300 and MI BCIs separately. 22 testing runs were considered in three blocks: (1) A block for hybrid control testing, (2) A block for MI-based navigation, and (3) A block for P300-based device control. Three tasks with a combination of navigation and device control commands were considered for evaluating the hybrid control strategy. In block presenting MI-based navigation, the tasks were the same, with the difference that in the device control state areas, the device control panels were not evoked. In the third block, navigation was not available and two of the tasks were tested for P300 BCI evaluation. The online accuracy was used for comparing different approaches. Comparing the P300 task in the hybrid BCI and the single P300 BCI showed little reduction in the accuracy of the hybrid strategy. The accuracy for two of the subjects reduced in MI part of the hybrid BCI compared to the single MI BCI. However, by utilizing the hybrid BCI, more complicated tasks can be accomplished in a virtual environment.

In [33], P300 and ERD were introduced to be components of the hybrid BCI in robotic control decision applications. Parallel and asynchronous classifications were introduced. The system task was to detect the intended pattern. Classification accuracy was evaluated during the experiment, which was considered for presenting the hybrid. Sixty trials were presented to four subjects: thirty trials for P300 presentation and thirty trials for MI. During the second thirty trials, the P300 stimuli were also presented but the subjects were not supposed to pay any attention to the stimuli.

3.4. EEG (MI-Based)-NIRS Hybrid BCI. A type of hybrid BCI that uses EEG and NIRS [41] was introduced by [34]. Coyle et al. [4] introduced an approach of utilizing NIRS as an optical BCI. In [34], EEG and NIRS measurements were utilized simultaneously for ERD-based BCIs. In this study, the experiment consisted of 2 blocks of motor execution and 2 blocks of motor imagery. For all blocks, both EEG and NIRS were measured simultaneously. The increase in concentration of oxygenated hemoglobins ( $\mathrm{HbO}$ ) and decrease in concentration of deoxygenated hemoglobins $(\mathrm{HbR})$ were measured using NIRS. The global peak cross-validation accuracy for each subject was considered for evaluation of the hybrid BCI. The mean classification accuracies of $\mathrm{HbO}, \mathrm{HbR}$, and EEG for executed movement tasks were $71.1 \%, 73.3 \%$, and $90.8 \%$. For motor imagery tasks they were $71.7 \%, 65.0 \%$, and $78.2 \%$. The mean classification accuracies of EEG/HbO, $\mathrm{EEG} / \mathrm{HbR}$, and $\mathrm{EEG} / \mathrm{HbO} / \mathrm{HbR}$ for executed movement tasks were $92.6 \%, 93.2 \%$, and $87.4 \%$, and for motor imagery tasks were $83.2 \%, 80.6 \%$, and $83.1 \%$, respectively. It was shown that the combination of EEG and NIRS improved the classification accuracy in both MI and executed movement tasks. However, the information transfer rate may decrease. This type of hybrid BCI may enhance the performance of subjects who are not able to use EEG-based BCI properly.

3.5. SSVEP-NIRS Hybrid BCI. The NIRS-based BCI was used as a brain switch for a SSVEP BCI system [26]. The objective was to open and close an orthosis. One subject with four runs performed an experiment. A $60 \mathrm{sec}$ break was considered between two runs. For starting a command, the optical BCI was utilized as a switch for SSVEP BCI starting point. By using a switch, false positives were detected during the first two runs, but in the third run, the performance was improved and only one false positive occurred. In the last run, the performance was perfect with $100 \%$ accuracy.

3.6. EEG-EMG Hybrid BCI. EEG and EMG were fused to devise a hybrid BCI in [35]. EEG signals were recorded through 16 channels. EMG activities were recorded from 
four channels over the flexor and extensor of the right and the left forearms. Two classifiers were used for EEG and EMG and the probabilities from these classifiers were used for controlling the BCI feedback. In the first approach of this experiment, a switch with weights equally balanced between the two classifiers was implemented between the input channels as the fusion of EEG and EMG. In the second approach, the Bayesian fusion method was utilized. Two conditions were considered for EEG and EMG separately and four conditions for the fusion of EEG and EMG depending on the increase of muscular fatigue. The accuracy for EEG activity alone was $73 \%$ and for EMG activity alone was $87 \%$. It was improved $91 \%$ in the hybrid BCI, in the first approach, the accuracy was $90 \%$ for $10 \%$ attenuation due to the fatigue, $85 \%$ for $50 \%$ attenuation, and $73 \%$ for $90 \%$ attenuation. Results had the same trend in the second approach with smaller standard deviation (SD). The accuracy was $92 \%$ for $50 \%$ attenuation, and $60.4 \%$ for $90 \%$ attenuation. In the third condition, the accuracy achieved was less than the accuracy in EEG BCI and this is because of the assumption of fixed value sources in the Bayesian fusion technique calculations. Utilizing multimodal fusion techniques led to enhancement in performance reliability.

3.7. EEG-EOG Hybrid BCI. Since the majority of people with disabilities can have control on their eye movement, the electrooculogram (EOG) signals could be an appropriate option as input signals for BCI system. EEG and EOG combination was introduced to make a hybrid BCI [36]. In this study, EOG and EEG signals were taken from two channels and were utilized simultaneously. The technique in generating control commands based on EEG/EOG hybrid BCI is explained as follows.

The "turn left" and "turn right" control commands were derived from EOG signals based on the right/left eye gazing pattern. Subjects performed maximum right and left eye gazing, and the positive and negative potential were recorded, respectively. $75 \%$ of the recorded amplitudes were considered as the threshold for detecting the right and left eye movements. If the amplitude recorded from the right eye during the trials was greater than the threshold related to the right eye, the "turn right" command was detected. For the "turn left" control command detection, the absolute value of the negative potential recorded from the left eye should be greater than the related threshold. If both values were less than the related threshold values, the "no action" control command was detected. Classification accuracy of 100\% was achieved for "turn left" and "turn right" control commands. Average accuracy of $95 \%$ was achieved for "no action" control command. The "forward", "no action", and "completely stop" control commands were detected from EEG. The parameter used for deriving the control commands from the EEG was PSD in the alpha and beta band. A threshold was considered for comparison to the maximum PSD detected from the alpha and beta band. Three subjects in three trials and 50 control commands in each trial performed experiments. At the beginning of the test, software calibration was performed by the subjects. The maximum PSD in alpha band was recorded from the subjects with closed eyes and 75\% of the PSD from the calibration was considered as threshold. For the "completely stop" command, the subjects were instructed to close their eyes to increase the alpha activity. Then, the maximum PSD in the alpha band was compared to a threshold. If the maximum PSD was greater than the threshold, the "completely stop" command was issued. For the "forward" control command, the subjects were instructed to think about moving forward. If the maximum PSD recorded in the beta band was greater than the maximum PSD recorded in the alpha band, the "forward" control was issued. The "no action" control command was issued if the maximum PSD in the beta band was less than the maximum PSD in the alpha band and both were less than the threshold. The average classification accuracy over the whole trials was $100 \%$ for "completely stop" and 87\% for "forward" control commands. The "no action" control command was common in both EEG and EOG control command detection parts and the average classification accuracy of $95 \%$ was related to the both parts of the task. The interface implementation and the feedback were presented by employing the test on a toy truck. In addition to high classification accuracy, small number of electrodes and short training time showed the advantage of introduced hybrid.

In another approach [37], a self-paced BCI system was combined with an eye-tracker system to establish a selfpaced hybrid BCI [26]. In this system, for cursor control, an eye tracker was utilized by detecting the user's eye gaze. A BCI was utilized for clicking on a selected item on computer screen. Subjects were instructed to first gaze at an intended letter on the screen to select it, then for click on the selected letter, hand extension movement was needed. EEG was recorded from the cortex area with 15 electrodes. For EOG, two pairs of electrodes were used. In addition, four pairs of electrodes were used for recording facial muscle activities, from which the facial muscle artefacts can be detected. PSD of 30 combinations of bipolar EEG channels was computed based on Fast Fourier Transform (FFT). For feature selection, stepwise LDA was considered [42]. Then, the features were classified with LDA and adaptive LDA and for more improvement moving average.

For removing EOG and EMG artefacts from the EEG signal, an algorithm was proposed in [43], which showed improvement in the performance of the introduced selfpaced hybrid BCI [37]. Stationary wavelet transform and an adaptive threshold mechanism were used in the proposed algorithm. Results were evaluated based on two types of data; real EEG signals with simulated artefacts (semi-simulated EEG signals) and real EEG signals. In semi-simulated EEG signals, signal distortion was decreased and in real EEG signals, the true positive rate was increased using the proposed algorithm.

\section{Conclusion}

To overcome limitations and disadvantages of conventional BCIs, different BCI systems or BCI and non-BCI systems can be combined to form a "hybrid BCI". In this paper, different methods of establishing a hybrid BCI system were discussed 
and compared. Hybrid BCIs have been used for different applications such as $2 \mathrm{D}$ control of a cursor, target selection, and virtual environment.

There are several advantages of sequential combination when one of the BCIs is used as a switch or different BCIs are used for different tasks sequentially. When combined sequentially, complicated tasks can be distributed to several stages in series. For each stage, a specific BCI can be used. An example of this approach is a virtual environment application [30]. Based on the required type of control commands, different BCI systems can be implemented. In [16], one BCI (ERD) was used as a switch for another BCI (SSVEP) and the false positive rate was decreased for this sequential hybrid BCI. However, the main advantage of the simultaneous combination is that in general the accuracy can be improved if the BCIs are combined appropriately for all subjects. With adaptive pattern recognition algorithms, a hybrid system can adapt to subjects based on their performance. In addition, classification methods can use more BCI outputs. Hybrid BCIs combining different systems simultaneously may be more complicated than a single BCI and more difficult to be accepted by all users. Therefore, the paradigm design of a hybrid BCI plays a very important role in the overall performance of the system. Similarly, when a BCI system is combined with a non-BCI, which is not based on EEG signals, the system performance can be improved. In general, in a hybrid BCI, the complexity of the system paradigm is increased compared to a non-hybrid BCI. Therefore, the use of hybrid systems might be more complicated from the user's point of view. Thus, in designing a hybrid system paradigm, the complexity and user acceptability are important performance criteria to be considered carefully. Another consideration for the user acceptability is the number of channels used in a hybrid BCI system.

In conclusion, although hybrid BCIs have shown great improvements in several performance criteria such as accuracy and information transfer rate, complexity of the system, and user acceptability should be reported as important performance criteria of hybrid BCI systems. With the current trend in introducing hybrid BCIs, we will soon see more than two BCI systems combined sequentially or simultaneously. It is also possible to combined BCIs in a combined sequentially/simultaneously approach. This will create a network of BCIs which cannot be distinguished as sequential or simultaneous any more. For example, for tasks with heavy object selections, SSVEP can be used for one stage and P300 for another stage. After the object selection, ERD/ERS BCI would be presented for continuous control tasks for other commands.

\section{References}

[1] J. R. Wolpaw, N. Birbaumer, D. J. McFarland, G. Pfurtscheller, and T. M. Vaughan, "Brain-computer interfaces for communication and control," Clinical Neurophysiology, vol. 113, no. 6, pp. 767-791, 2002.

[2] N. Weiskopf, R. Veit, M. Erb et al., "Physiological self-regulation of regional brain activity using real-time functional magnetic resonance imaging (fMRI): methodology and exemplary data," NeuroImage, vol. 19, no. 3, pp. 577-586, 2003.

[3] S. Waldert, H. Preissl, E. Demandt et al., "Hand movement direction decoded from MEG and EEG," Journal of Neuroscience, vol. 28, no. 4, pp. 1000-1008, 2008.

[4] S. Coyle, T. Ward, C. Markham, and G. McDarby, "On the suitability of near-infrared (NIR) systems for next-generation brain-computer interfaces," Physiological Measurement, vol. 25, no. 4, pp. 815-822, 2004.

[5] N. Birbaumer, N. Ghanayim, T. Hinterberger et al., "A spelling device for the paralysed," Nature, vol. 398, no. 6725, pp. 297-298, 1999.

[6] E. E. Sutter, "The brain response interface: communication through visually-induced electrical brain responses," Journal of Microcomputer Applications, vol. 15, no. 1, pp. 31-45, 1992.

[7] J. J. Vidal, "Toward direct brain-computer communication," Annual Review of Biophysics and Bioengineering, vol. 2, pp. 157$180,1973$.

[8] J. J. Vidal, "Real-time detection of brain events in EEG," Proceedings of the IEEE, vol. 65, no. 5, pp. 633-641, 1977.

[9] B. Allison, J. Faller, and C. H. Neuper, "BCIs that use steady-state visual evoked potentials or slow cortical potentials," in BrainComputer Interfaces: Principles and Practice, Wolpaw and E. W. Wolpaw, Eds., Oxford University Press, 2012.

[10] E. Sellers, Y. Arbel, and E. Donchin, "BCIs that uses P300 eventrelated potentials," in Brain-Computer Interfaces: Principles and Practice, J. Wolpaw and E. W. Wolpaw, Eds., Oxford University Press, 2012.

[11] J. Kalcher, D. Flotzinger, C. Neuper, S. Gölly, and G. Pfurtscheller, "Graz brain-computer interface II: towards communication between humans and computers based on online classification of three different EEG patterns," Medical and Biological Engineering and Computing, vol. 34, no. 5, pp. 382-388, 1996.

[12] B. Z. Allison, E. W. Wolpaw, and J. R. Wolpaw, "Brain-computer interface systems: progress and prospects," Expert Review of Medical Devices, vol. 4, no. 4, pp. 463-474, 2007.

[13] L. A. Farwell and E. Donchin, "Talking off the top of your head: toward a mental prosthesis utilizing event-related brain potentials," Electroencephalography and Clinical Neurophysiology, vol. 70, no. 6, pp. 510-523, 1988.

[14] C. Brunner, B. Z. Allison, D. J. Krusienski et al., "Improved signal processing approaches in an offline simulation of a hybrid brain-computer interface," Journal of Neuroscience Methods, vol. 188, no. 1, pp. 165-173, 2010.

[15] B. Z. Allison, C. Brunner, V. Kaiser, G. R. Müller-Putz, C. Neuper, and G. Pfurtscheller, "Toward a hybrid brain-computer interface based on imagined movement and visual attention," Journal of Neural Engineering, vol. 7, no. 2, Article ID 026007, 2010.

[16] G. Pfurtscheller, T. Solis-Escalante, R. Ortner, P. Linortner, and G. R. Muller-Putz, "Self-paced operation of an SSVEP-based orthosis with and without an imagery-based "brain switch": a feasibility study towards a hybrid BCI," IEEE Transactions on Neural Systems and Rehabilitation Engineering, vol. 18, no. 4, pp. 409-414, 2010.

[17] B. Allison, T. Luth, D. Valbuena, A. Teymourian, I. Volosyak, and A. Graser, "BCI demographics: How many (and what kind of) people can use a SSVEP BCI?" IEEE Transactions on Neural Systems and Rehabilitation Engineering, vol. 18, no. 2, pp. 107116, 2010. 
[18] E. E. Sutter, "The visual evoked response as a communication channel," in Proceedings of the Symposium on Biosensors, pp. 95100, 1984.

[19] Y. Wang, Y. T. Wang, and T. P. Jung, "Visual stimulus design for high-rate SSVEP BCI," Electronics Letters, vol. 46, no. 15, pp. 1057-1058, 2010.

[20] R. Fazel-Rezai, B. Z. Allison, C. Guger, E. W. Sellers, S. C. Kleih, and A. Kübler, "P300 brain computer interface: current challenges and emerging trends," Frontiers in Neuroengineering, vol. 5, 2012.

[21] E. Donchin and M. G. Coles, "Is the P300 component a manifestation of context updating?" Behavioral and Brain Functions, vol. 11, pp. 357-374, 1998.

[22] W. Lutzenberger, T. Elbert, B. Rockstroh, and N. Birbaumer, "The effects of self-regulation of slow control potentials on performance in a signal detection task," International Journal of Neuroscience, vol. 9, no. 3, pp. 175-183, 1979.

[23] A. Kübler, B. Kotchoubey, T. Hinterberger et al., "The thought translation device: a neurophysiological approach to communication in total motor paralysis," Experimental Brain Research, vol. 124, no. 2, pp. 223-232, 1999.

[24] G. Pfurtscheller and F. H. Lopes Da Silva, "Event-related EEG/MEG synchronization and desynchronization: basic principles," Clinical Neurophysiology, vol. 110, no. 11, pp. 1842-1857, 1999.

[25] http://www.scopus.com/home.url.

[26] G. Pfurtscheller, B. Z. Allison, C. Brunner et al., "The hybrid BCI," Frontiers in Neuroscience, vol. 4, 2010.

[27] A. Savic, U. Kisic, and M. Popovic, "Toward a hybrid BCI for grasp rehabilitation," in Proceedings of the 5th European Conference of the International Federation for Medical and Biological Engineering Proceedings, pp. 806-809, 2012.

[28] C. Brunner, B. Z. Allison, C. Altstätter, and C. Neuper, "A comparison of three brain-computer interfaces based on eventrelated desynchronization, steady state visual evoked potentials, or a hybrid approach using both signals," Journal of Neural Engineering, vol. 8, no. 2, Article ID 025010, 2011.

[29] R. C. Panicker, S. Puthusserypady, and Y. Sun, "An asynchronous P300 BCI with SSVEP-based control state detection," IEEE Transactions on Biomedical Engineering, vol. 58, no. 6, pp. 1781-1788, 2011.

[30] G. Edlinger, C. Holzner, and C. Guger, "A hybrid braincomputer interface for smart home control," in Proceedings of the 14th international conference on Human-Computer Interaction. Interaction Techniques and Environments, pp. 417-426, 2011.

[31] B. Rebsamen, E. Burdet, Q. Zeng et al., "Hybrid P300 and MuBeta brain computer interface to operate a brain controlled wheelchair," in Proceedings of the 2nd International Convention on Rehabilitation Engineering and Assistive Technology, pp. 5155, 2008.

[32] Y. Su, Y. Qi, J. X. Luo et al., "A hybrid brain-computer interface control strategy in a virtual environment," Journal of Zhejiang University, vol. 12, no. 5, pp. 351-361, 2011.

[33] H. Riechmann, N. Hachmeister, H. Ritter, and A. Finke, "Asynchronous, parallel on-line classification of P300 and ERD for an efficient hybrid BCI," in Proceedings of the 5th International IEEE/EMBS Conference on Neural Engineering (NER '11), pp. 412-415, May 2011.

[34] S. Fazli, J. Mehnert, J. Steinbrink et al., "Enhanced performance by a hybrid NIRS-EEG brain computer interface," Neuroimage, vol. 59, pp. 519-529, 2011.
[35] R. Leeb, H. Sagha, R. Chavarriaga, and J. D. R. Millán, “A hybrid brain-computer interface based on the fusion of electroencephalographic and electromyographic activities," Journal of Neural Engineering, vol. 8, no. 2, Article ID 025011, 2011.

[36] Y. Punsawad, Y. Wongsawat, and M. Parnichkun, "Hybrid EEGEOG brain-computer interface system for practical machine control," in Proceedings of the IEEE Engineering in Medicine and Biology Society Conference (EMBC '10), pp. 1360-1363, 2010.

[37] X. Yong, M. Fatourechi, R. K. Ward, and G. E. Birch, "The design of a point-and-click system by integrating a self-paced brain-computer interface with an eye-tracker," IEEE Journal on Emerging and Selected Topics in Circuits and Systems, vol. 1, no. 4, pp. 590-602, 2011.

[38] A. Kubler and K. R. Muller, "An introduction to brain-computer interfacing," in Toward Brain-Computer Interfacing, G. Dornhedge, J. R. Millan, T. Hinterberger, D. J. McFarland, and K. R. Muller, Eds., pp. 1-25, MIT Press, Cambridge, Mass, USA, 2007.

[39] D. J. Krusienski, E. W. Sellers, F. Cabestaing et al., "A comparison of classification techniques for the P300 Speller," Journal of Neural Engineering, vol. 3, no. 4, article 299, 2006.

[40] U. Hoffmann, J. M. Vesin, T. Ebrahimi, and K. Diserens, "An efficient P300-based brain-computer interface for disabled subjects," Journal of Neuroscience Methods, vol. 167, no. 1, pp. 115-125, 2008.

[41] T. Tsubone, T. Muroga, and Y. Wada, "Application to robot control using brain function measurement by near-infrared spectroscopy," in Proceedings of the 29th IEEE Engineering in Medicine and Biology Society (EMBC '07), pp. 5342-5345, August 2007.

[42] P. A. Lachenbruch and M. Goldstein, "Discriminant analysis," Biometrics, vol. 35, no. 1, pp. 69-85, 1979.

[43] X. Yong, M. Fatourechi, R. K. Ward, and G. E. Birch, "Automatic artefact removal in a self-paced hybrid brain-computer interface system," Journal of NeuroEngineering and Rehabilitation, vol. 9, article 50, 2012. 

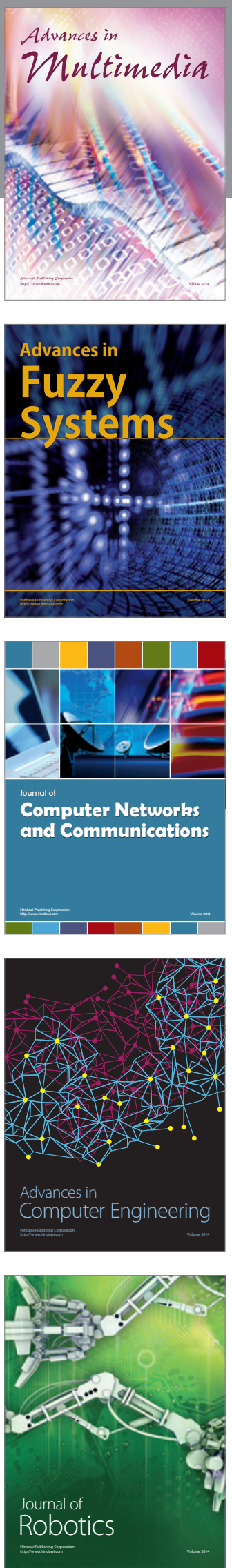

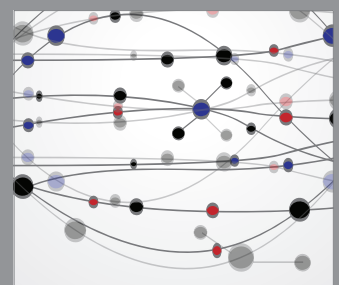

The Scientific World Journal
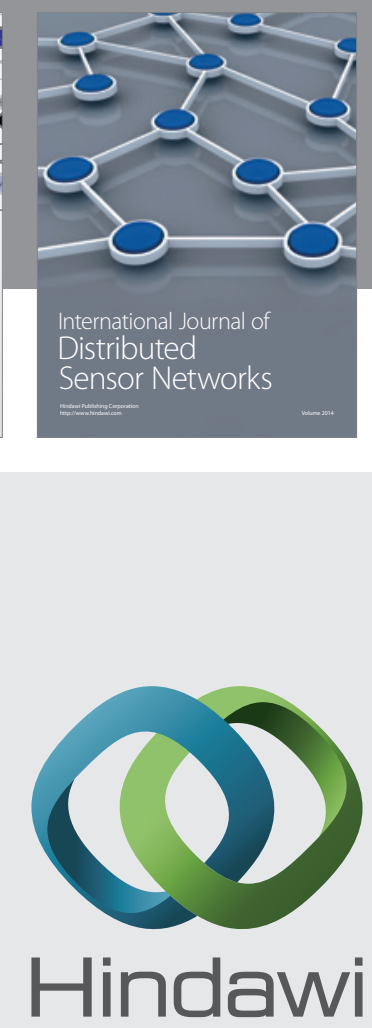

Submit your manuscripts at

http://www.hindawi.com
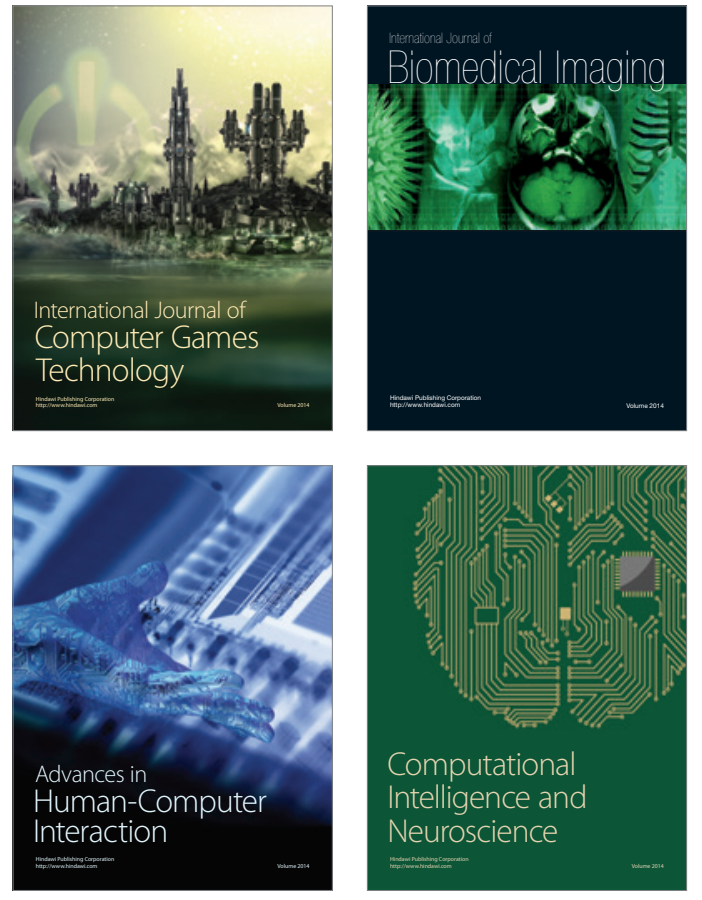
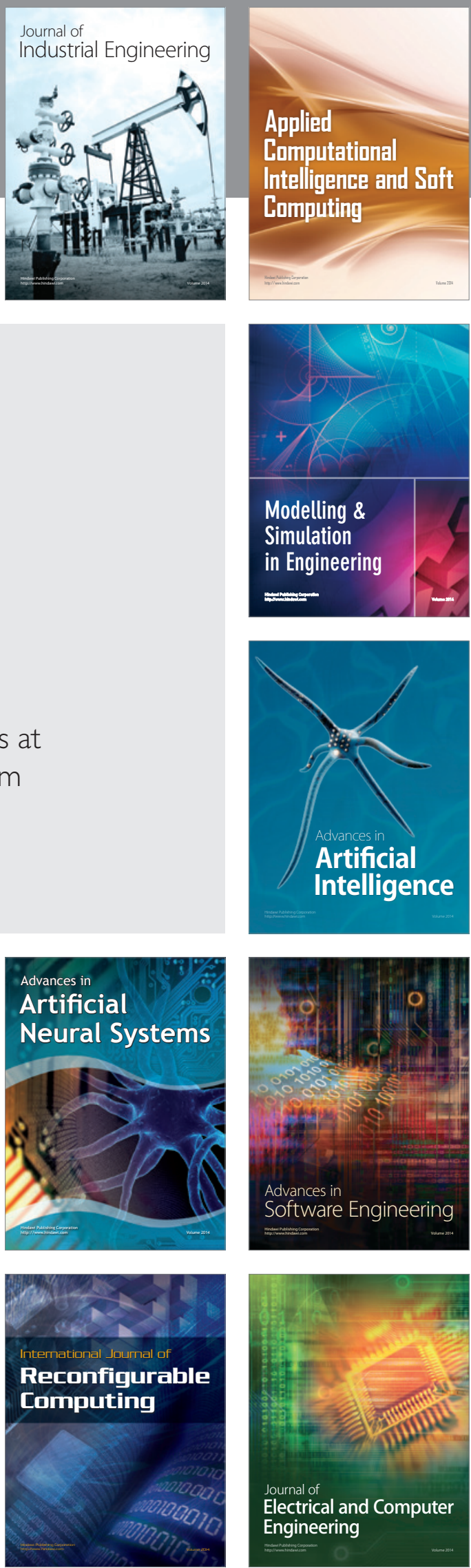